\title{
Environmental modifiable risk factors for multiple sclerosis: Report from the 2016 ECTRIMS focused workshop
}

\author{
Maria Pia Amato, Tobias Derfuss, Bernard Hemmer, Roland Liblau, Xavier Montalban, \\ Per Soelberg Sørensen and David H Miller; For the 2016 ECTRIMS Focused Workshop Group*
}

\begin{abstract}
Multiple sclerosis (MS) is an inflammatory and neurodegenerative demyelinating disease of the central nervous system (CNS), most likely autoimmune in origin, usually beginning in early adulthood. The aetiology of the disease is not well understood; it is viewed currently as a multifactorial disease which results from complex interactions between genetic predisposition and environmental factors, of which a few are potentially modifiable. Improving our understanding of these factors can lead to new and more effective approaches to patient counselling and, possibly, prevention and management of the disease. The 2016 focused workshop of the European Committee for Treatment and Research in Multiple Sclerosis (ECTRIMS) addressed the topic of environmental, modifiable risk factors for MS, gathering experts from around the world, to collate experimental and clinical research into environmental factors that have been associated with the disease onset and, in a few cases, disease activity and progression. A number of factors, including infections, vitamin D deficiency, diet and lifestyle factors, stress and comorbidities, were discussed. The meeting provided a forum to analyse available evidence, to identify inconsistencies and gaps in current knowledge and to suggest avenues for future research.
\end{abstract}

Keywords: Multiple sclerosis, environmental risk factors, infections, vitamin D, lifestyle, comorbidities

Date received: 7 November 2016; revised: 7 December 2016; accepted: 7 December 2016

\section{Introduction}

Multiple sclerosis (MS) is an inflammatory and neurodegenerative demyelinating disease of the central nervous system (CNS) with onset usually in young adulthood; it is likely autoimmune in origin. ${ }^{1}$ Prevalence and incidence rates vary with geography and ethnicity, with prevalence figures ranging from 2 per 100,000 in Japan to greater than 100 per 100,000 in Northern Europe and North America. Women are affected two to three times more frequently than men, ${ }^{2}$ and there is evidence that this disparity is increasing in some, but not all, geographical areas. ${ }^{3} \mathrm{MS}$ is usually diagnosed during the most active and productive period of life in the majority of individuals, with disability eventually developing in a sizeable proportion of patients. There is therefore a compelling need to understand whether modifiable factors may alter disease risk, as well as disease activity and progression.

The aetiology of MS is most certainly heterogeneous: multiple factors, both genetic and environmental, determine disease risk and these risk factors interact with one another in a complex manner. ${ }^{4}$ The strongest contributor to genetic susceptibility is the major histocompatibility complex (MHC). The HLADRB1*1501-DRB5*0101 haplotype, in particular, shows a strong association with MS risk in Caucasians. ${ }^{5}$ Many other susceptibility gene variants have now been identified, although their effects are generally minor. 6,7 The genetics of the HLA region, however, is complex with potential epistatic interactions between DR haplotypes and heterogeneity depending on ethnicity. ${ }^{8}$ Notwithstanding this, for a substantial number of people who are genetically susceptible to MS, it does appear that non-genetic, potentially modifiable factors play a role in whether they develop the disease or not. In addition, some modifiable factors have been identified which are associated with disease activity and progression. ${ }^{9}$ Together, this suggests that interventions could be developed to prevent some proportion of MS cases and/or alleviate the severity of the disease course.
Multiple Sclerosis Journal 2018, Vol. 24(5) 590-603 DOI: 10.1177 1352458516686847

(C) The Author(s), 2017. Reprints and permissions: http://www.sagepub.co.uk/ journalsPermissions.nav
Correspondence to: MP Amato Department of NEUROFARBA, Section Neurosciences, University of Florence, Largo Bramabilla 3 , Florence 50134, Italy. mariapia.amato@unifi.it

Maria Pia Amato Department of NEUROFARBA, Section Neurosciences, University of Florence, Florence, Italy

Tobias Derfuss Departments of Neurology and Biomedicine, University Hospital Basel, Basel, Switzerland

Bernard Hemme Technische Universitat Munchen, Munchen, Germany

Roland Liblau Faculte de Medecine Purpan, Universite Toulouse III - Paul Sabatier, Toulouse, France

Xavier Montalban Unitat de Neuroimmunologia Clínica, Barcelona, Spain Per Soelberg Sørensen Det Sundhedsvidenskabelige Fakultet, Kobenhavns Universitet, Kobenhavn, Denmark

David H Miller Queen Square MS Centre, Department of Neuroinflammation, UCL Institute of Neurology, University College London, London, UK

*The members of ECTRIMS Focused Workshop Group are listed in Appendix 1. 
ECTRIMS held its fourth focused workshop on environmental, modifiable risk factors for MS on 3-4 March 2016 in Florence, Italy. The purpose of this report is to summarise the highlights from this meeting and their implications for patient care and to identify avenues for future research that may generate more effective approaches to prevention and management of MS.

\section{Gene-environment interactions}

More than 110 loci have been associated with MS onset in large, multicentre, genome-wide association studies, although the strongest association remains with the HLA-DRB1*1501-DRB5*0101 haplotype. 6,7 Epidemiological studies have aimed to identify causal associations between gene-environment interactions or environment-environment interactions and MS risk. ${ }^{4}$

Among various research initiatives, the Swedish MS Registry has launched the development of the Epidemiology in Multiple Sclerosis (EIMS) and Genes and Environment in Multiple Sclerosis (GEMS) projects. $^{10}$ Lifestyle questionnaires and extensive genotyping of subjects included in these cohort studies have enabled identification of environmental and lifestyle MS risk factors and their possible interaction. The studies have shown interactions between MS HLA risk genes and smoking (both active and passive), Epstein-Barr virus (EBV) serology, infectious mononucleosis (IM), adolescent obesity and organic solvents. It is noteworthy that in the registry dataset $60 \%$ of all cases were associated with an interaction between EBV serology and HLA, with an additive effect.

In general, whether MS risk factors act in sequence and depend on each other or whether they act independently and in an additive or multiplicative fashion is poorly understood. The fact that no factor has yet been shown to be present in all patients with MS, with the possible exception of the EBV in adult-onset MS, suggests that causal pathways are likely to differ between individuals and would support an additive effect. ${ }^{11}$

\section{Infections and vaccines}

Research on infectious causes of MS is based on clinical and pathological observations or experiments in animal models. ${ }^{12}$ Another approach involves the assessment of the extent to which an infectious agent could be implicated in the epidemiology of MS. ${ }^{13}$ In particular, the potential relationships between MS epidemiology and infections have been analysed in the context of the 'hygiene' and 'old friends' hypotheses (see below). A common aspect to both is that a widespread microbe, rather than a rare pathogen, may be involved in MS. Overall, epidemiological data provide some support to the 'hygiene' hypothesis, but with the additional proviso for a key role of EBV in determining MS risk.

A correlation between 'hygiene', as defined by reduced early life exposure to infectious agents, and MS risk has long been noted. It has been suggested that better hygiene or lower exposure to infectious agents in childhood can favour the development of a Th1 pro-inflammatory cellular immune response, whereas exposure to multiple infections in childhood could deviate the immune response towards a regulatory T-cell phenotype. ${ }^{14}$

The 'hygiene hypothesis' may explain some epidemiological findings, including a lower incidence of MS in developing countries (with poorer hygiene) as compared to countries with higher socioeconomic standards, and an increased MS prevalence associated with improving education and socioeconomic status (SES) in some early investigations, ${ }^{14}$ although more recently the inconsistency of data surrounding SES and MS risk has been recognized. ${ }^{15}$ This hypothesis is also corroborated by the observation of an increased risk of MS with a late age at infection with EBV and manifestation as IM. ${ }^{16}$ However, the hypothesis does not completely fit with epidemiological MS data, since those subjects who are seronegative for EBV infection have a very low MS risk status. This represents a 'paradox': if the hygiene hypothesis was valid for MS, these individuals would be expected to be at high MS risk because the lack of infection from EBV (an almost ubiquitous pathogen) is a marker of a highly hygienic environmental status. ${ }^{14}$ This paradox could be solved if EBV itself is a contributory cause of MS - that is, the adverse effect of good hygiene on MS risk becomes manifest only after EBV infection.

\section{$E B V$}

The identification of the paradox described above, and the demonstration that EBV-seronegative individuals are virtually immune to MS until the time they are infected with EBV but are at high MS risk after EBV infection, ${ }^{17}$ provides convincing evidence that EBV stands out as the most important infectious agent that may explain many of the key features of MS epidemiology.

Furthermore, EBV and IM (which is usually caused by primary EBV infection during adolescence) have 
been linked to MS onset in several epidemiological studies, both retrospective and prospective in design, and in meta-analyses and basic research. A number of EBV-associated autoimmune disorders and malignancies may develop after an extended period of latency following primary infection. In particular, a history of IM is associated with a two- to threefold increased risk of developing MS. Moreover, an immune response to the $\mathrm{CD}^{+}{ }^{+}$T-cell antigen EBNA1 is prominently elevated in MS subjects, and titres of antiEBNA1 antibodies appear to increase the risk of the disease in a dose-dependent manner. ${ }^{14}$

However, the link between EBV and MS by itself cannot explain the decline in risk among migrants from high to low MS prevalence areas. ${ }^{13,18}$ This decline may suggest that either EBV strains in lowrisk areas have less propensity to cause MS or that other infectious or non-infectious factors modify the host response to EBV or contribute to determine MS risk. ${ }^{14}$

Proposed mechanisms of action for the association of EBV with MS include molecular mimicry, bystander damage to the CNS by EBV-specific T cells, induction of an immune response to EBNA1 antigen, chronic infection of B cells and an indirect mechanism due to reactivation of human endogenous retroviruses (HERV), such as HERV-W. ${ }^{14}$

In conclusion, while overall evidence strongly supports a role for EBV in MS aetiology, the exact mechanism by which EBV infection can predispose to MS remains elusive. Further studies are strongly advocated to elucidate the nature of this association. In addition, further work should investigate whether EBV infection can also drive subsequent disease activity or progression in those with established MS. ${ }^{9}$

\section{Implications for prevention and patient care}

Based on the above evidence on the role of EBV in MS, there could be relevant implications for therapeutic/preventive strategies. Antiviral therapy, based on nucleoside analogues, has been studied principally in EBV-related lymphoproliferative disorders, but it is generally ineffective, probably because the virus is in a latent phase of its cycle. Research into the development of prophylactic and therapeutic EBV vaccines has started. EBV vaccination, initially targeting IM, is under investigation, whereas induction of a viral neutralisation antibody response preventing primary infection may be appropriate for the prevention of primary EBV infection in the general population. An alternative strategy to induce a cell-mediated immunity to control latent-infected cells in persistently infected individuals may have a role in the prevention of EBV-related malignancies and autoimmune disorders. ${ }^{19}$

Due to the capability of the virus to establish latent infection in B cells, ${ }^{14}$ in those who already have MS, potential therapeutic strategies include B-celldepleting therapies and anti-retroviral drugs. Rituximab, ocrelizumab and ofatumumab are monoclonal antibodies targeting $\mathrm{CD} 20^{+} \mathrm{B}$ cells which have shown disease-ameliorating effects in clinical trials. ${ }^{20}$ The role of HERVs in MS pathogenesis is under investigation, and agents such as raltegravir, a retroviral integrase inhibitor, have been tested in clinical trials for the treatment of MS. ${ }^{21,22}$

The Bacillus Calmette-Guérin (BCG) vaccine is under study as a possible option for an 'intermediate' preventive strategy, based on vaccination of those who are at high risk of developing MS. In a randomised double-blind trial on subjects with clinically isolated syndrome (CIS), BCG vaccine, as compared to placebo, was associated with significantly reduced development of gadolinium-enhancing lesions at magnetic resonance imaging (MRI) for a 6-month period before starting immunomodulating therapy. ${ }^{23} \mathrm{BCG}$ vaccine may act as a tumour necrosis factor (TNF)-inducer, and preclinical studies have shown that it may have a neuroprotective effect. ${ }^{23} \mathrm{~A}$ study with BCG vaccine in subjects with radiologically isolated syndromes (RIS) is in preparation (Marco Salvetti, MD, University of Rome, personal communication).

\section{Parasites}

The 'old friend' hypothesis suggests that early exposure to large numbers of parasites could protect against development of MS in later life. ${ }^{24}$ Indeed, the risk of MS shows an inverse correlation with helminth infections, and it is possible that helminths - which have been parasitic throughout human evolution - act as immune regulators in people naturally infected with them. Helminths can establish a life-long equilibrium with the host, favouring the release of antiinflammatory cytokines and expansion of regulatory $\mathrm{T}$ and $\mathrm{B}$ cells. There are some positive results with the use of helminths as a potential treatment in animal models of MS. A few preliminary clinical studies with helminth therapy have been conducted in MS patients. ${ }^{24}$ A small controlled trial of Trichuris suis ova failed to show any benefit. ${ }^{25}$ Future research should focus on the host-parasite interactions resulting from specific molecules isolated from parasites, 
perhaps providing a more targeted approach and minimising adverse effects related to helminth therapy.

\section{Vitamin D deficiency}

Vitamin D and MS risk

A substantial evidence base now exists to support an association between vitamin D deficiency and low levels of its metabolite 25-hydroxyvitamin $\mathrm{D}_{3}$ $(25(\mathrm{OH}) \mathrm{D})$ in the onset and development of MS. ${ }^{26}$ Experimental studies have shown that vitamin D has potent immunomodulatory activity. ${ }^{26}$ It may have an anti-inflammatory role in the secondary lymphoid organs and the CNS, and it may also have neuro- and myelin-protective and/or myelin-regenerative effects in the CNS. ${ }^{27}$ Genetic effects may also contribute to MS risk as genes associated with vitamin D metabolism have been identified in an MS genome association study. In addition, transcription of the HLA-DRB $1 * 1501$ locus is regulated by vitamin D. ${ }^{28}$

The bulk of evidence for this association is derived from extensive epidemiological research based on different study designs. Collectively, the results support the importance of vitamin D sufficiency in adolescence and adulthood, up to the time of MS onset. Furthermore, they also suggest the importance of vitamin D in childhood or even in utero. ${ }^{14}$ Recently, the role of maternal vitamin $\mathrm{D}$ deficiency during pregnancy has been documented in a nested casecontrol study by the Finnish Maternity Cohort, ${ }^{29}$ where insufficient maternal vitamin D levels during pregnancy were associated with an almost twofold increased risk of MS in the offspring.

The causality of the relationship between vitamin $\mathrm{D}$ and MS risk is further supported by the results of Mendelian randomisation studies. These results provide compelling evidence that vitamin D deficiency is an independent risk factor for MS, although they do not exclude a contribution of other factors. Some uncertainty remains about whether there is a critical timing of exposure to low 25(OH)D levels and what absolute level of $25(\mathrm{OH}) \mathrm{D}$ is associated with increased MS risk. ${ }^{30,31}$

\section{Vitamin D and MS disease activity or progression} Vitamin D deficiency has also been associated with increased disease activity or progression in subjects with already established disease. The findings of a few small therapeutic trials using vitamin D in MS patients have been inconsistent, ${ }^{32-37}$ and none of these studies was powered sufficiently to observe a treatment effect. Large randomised interventional clinical trials in the United States, Europe and Australasia are currently evaluating vitamin D supplementation to decrease the risk of relapses (Table 1). These studies should also help to define appropriate methods of dosing to obtain optimal serum vitamin D levels that may reduce clinical MS activity.

Finally, observational studies based on the datasets from Phase III trials of interferon beta-1b support an influence of vitamin D on the disease course. In the betaferon/betaseron in newly emerging MS for initial treatment (BENEFIT) trial, 25(OH)D levels in the first year of the study were studied in association with clinical and MRI outcomes after 5 years, which helped minimise the possibility of reverse causation. ${ }^{42}$ The study results showed that higher serum $25(\mathrm{OH}) \mathrm{D}$ levels early in the course of MS robustly predicted a lower degree of clinical and MS activity, MRI lesion load, brain atrophy and clinical progression over the 5 years of follow-up. In the large cohort from the betaferon/betaseron efficacy yielding outcomes of a new dose in MS patients (BEYOND) trial in relapsing-remitting multiple sclerosis (RRMS), sub-group analyses based on the patient vitamin D status were possible. ${ }^{43}$ There were profound geographical differences in patient vitamin D status; the analyses showed a decreased risk of accumulating new active lesions at MRI in subjects with higher levels of 25(OH)D and suggested as 'best guess' an optimal level of $25(\mathrm{OH})$ $\mathrm{D}$ corresponding to $100 \mathrm{nmol} / \mathrm{L}$. In this regard, further investigations are needed to identify definitively optimal levels of vitamin D and to determine whether these results apply to different races or ethnicities, to patients with the secondary or primary progressive course of MS and to patients receiving drugs other than interferon beta- $1 b$.

\section{Implications for prevention and patient care}

Questions remain as to whether the MS incidence or ongoing disease activity and progression can be reduced by vitamin D supplementation. Available data suggest that MS risk is reduced at an average serum $25(\mathrm{OH}) \mathrm{D}$ level above $100 \mathrm{nmol} / \mathrm{L}$, currently achieved by only one-fifth of the at-risk population in the United States, and in a smaller proportion in most at-risk European populations. ${ }^{14}$ This level of vitamin D could be reached by taking $2000-4000$ IU/day of vitamin - a dose that is well above current recommendations, but has not been associated with major adverse outcomes to date. ${ }^{14}$

Due to the above uncertainties, many experts believe that more conclusive experimental evidence should 
Table 1. Ongoing randomised clinical trials of vitamin D in MS patients.

\begin{tabular}{|c|c|c|c|c|c|c|}
\hline Trial name & SOLAR $^{38}$ & VIDAMS ${ }^{39}$ & VITADEM & EVIDIMS $^{40}$ & PrevANZ ${ }^{41}$ & D-Lay-MS \\
\hline Location & Europe & USA & Spain & Germany & Australasia & France \\
\hline Participants & 230 RRMS & 172 RRMS & 100 RRMS & 80 RRMS or CIS & 240 CIS & 316 CIS \\
\hline $\begin{array}{l}\text { Vitamin D3 } \\
\text { intervention }\end{array}$ & $\begin{array}{l}7000 \mathrm{IU} / \text { day } \\
4 \text { weeks up to } \\
14,000 \mathrm{IU} / \text { day } \\
92 \text { weeks vs } \\
\text { placebo (add on to } \\
\text { IFNB-1a) }\end{array}$ & $\begin{array}{l}5000 \text { vs } 600 \mathrm{IU} / \\
\text { day (add on to } \\
\text { galtiramer acetate) }\end{array}$ & $\begin{array}{l}4000 \mathrm{IU} / \text { day vs } \\
\text { placebo }\end{array}$ & $\begin{array}{l}20,400 \text { IU/every other } \\
\text { day vs } 400 \text { IU/every } \\
\text { other day (add on to } \\
\text { IFNB-1b) }\end{array}$ & $\begin{array}{l}1000 \text { vs } 5000 \\
\text { vs } 10,000 \mathrm{IU} / \\
\text { day vs placebo }\end{array}$ & $\begin{array}{l}100,000 \mathrm{IU} / \\
\text { every } 14 \text { days vs } \\
\text { placebo }\end{array}$ \\
\hline Duration & 96 weeks & 96 weeks & Not known & 18 months & 48 weeks & 2 years \\
\hline $\begin{array}{l}\text { Primary } \\
\text { outcomes }\end{array}$ & $\begin{array}{l}\text { Mean number } \\
\text { CUA lesions } \\
\text { at week } 48 ; \\
\text { proportion relapse } \\
\text { free at the end of } \\
\text { study }\end{array}$ & $\begin{array}{l}\text { Percentage of } \\
\text { participants having } \\
\text { relapse by EOS }\end{array}$ & $\begin{array}{l}\text { Percentage of } \\
\text { participants } \\
\text { having relapse } \\
\text { by EOS }\end{array}$ & $\begin{array}{l}\text { Number of new } \mathrm{T} 2 \\
\text { lesions }\end{array}$ & $\begin{array}{l}\text { Conversion to } \\
\text { CDMS/MDMS }\end{array}$ & $\begin{array}{l}\text { Conversion to } \\
\text { CDMS/MDMS }\end{array}$ \\
\hline $\begin{array}{l}\text { Secondary } \\
\text { outcomes }\end{array}$ & $\begin{array}{l}\text { Annualised relapse } \\
\text { rate, EDSS change } \\
\text { at predefined time } \\
\text { points }\end{array}$ & $\begin{array}{l}\text { Annualised } \\
\text { relapse rate, EDSS } \\
\text { change, number of } \\
\text { new active lesions, } \\
\text { lesion volume }\end{array}$ & $\begin{array}{l}\text { EDSS change, } \\
\text { number of new } \\
\text { active lesions, } \\
\text { number of new } \\
\text { T2 hyperintense } \\
\text { lesions }\end{array}$ & $\begin{array}{l}\text { Annualised relapse } \\
\text { rate, percentage of } \\
\text { relapse free at EOS, } \\
\text { EDSS and MSFC, } \\
\text { change at predefined } \\
\text { time points }\end{array}$ & & $\begin{array}{l}\text { Number of } \\
\text { relapses, number } \\
\text { of new lesions, } \\
\text { new active } \\
\text { lesions, EDSS } \\
\text { change, EQ5D }\end{array}$ \\
\hline End of study & 2015 & 2016 & Not known & 2016 & Not known & 2017 \\
\hline
\end{tabular}

be awaited before implementing nationwide universal vitamin D supplementation programmes aimed at reducing the risk of new cases of MS. However, for many individuals, including those living in areas where vitamin D deficiency is highly prevalent and those at high MS risk due to their family history, in addition to CIS and MS patients, the use of vitamin D supplements may represent a reasonable choice, considering, on the one hand, the strength and quality of evidence available and, on the other hand, the low risk of adverse events using the vitamin D dose suggested above.

\section{Lifestyle risk factors}

\section{Cigarette smoking}

Cigarette smoking and MS risk. There is extensive and robust evidence that cigarette smoking, both active and passive, is a risk factor for MS onset. Smoking increases the risk of MS by approximately $50 \%$ in a number of epidemiological studies, ${ }^{44}$ and there appears to be a consistent dose-response relationship with cumulative smoking dose. ${ }^{45,46}$ Elevated levels of cotinine (a surrogate marker for nicotine) were detected in blood samples taken before the development of MS, supporting epidemiological evidence for the increased risk of MS in individuals who smoke. ${ }^{46}$ Differential changes over time in smoking behaviour between men (with decreasing numbers of smokers) and women (with a relatively constant number of smokers) over the past several decades could, in part, explain the increase in the female:male ratio in MS incidence noted in a few regions. ${ }^{14,47}$ The critical period for exposure to cigarette smoke is unclear, with some studies detecting no effect of age, ${ }^{45}$ and others observing increased susceptibility to MS with exposure during adolescence or young adulthood. ${ }^{46}$

Cigarette smoking and MS disease activity or progression. There is also some evidence of negative effects of smoking on disease course and progression. Smoking has been associated with a faster transition from onset of symptoms to a definitive MS diagnosis ${ }^{48}$ a more rapid shift to the secondary progressive phase of the disease, and progression in disability. ${ }^{49,50}$

Numerous mechanisms have been proposed to explain the adverse effects of smoking on MS risk, including effects on demyelination, disruption of the bloodbrain barrier, immunological effects and increased nitric oxide and nitric oxide metabolites, but all remain speculative. The observation that tobacco 
smoking, but not Swedish tobacco snuff, was associated with an increased MS risk suggests that combustion and/or inhalation are required to increase the risk of MS. Moreover, the increased risk of MS associated with organic solvents ${ }^{51}$ was found to be higher in never smokers, suggesting lung irritation may play a crucial role, ${ }^{52}$ which is also supported by studies in animal models..$^{53}$

Implications for prevention and patient care. Based on the above data, from a public health perspective, the impact of smoking and passive smoking on both MS onset and subsequent progression appears considerable. Promoting smoking cessation may be one of the most straightforward interventions available to reduce the incidence of MS.

Individuals with a history of MS in the family should be informed regarding the impact of smoking on the risk of developing MS, and the importance of preventing their children from being exposed to passive smoke.

Given the potential prognostic implications, patients with MS should be advised to stop smoking, to lessen risks for comorbidities and to avoid possible aggravating MS-related disability.

\section{Body mass index}

Body mass index and MS risk. In epidemiological studies, overweight/obesity is associated with a two- to threefold increased risk of MS. ${ }^{54-59}$ An interaction with other risk factors, including vitamin D status and genetic predisposition, has also been documented. Data from the Swedish EIMS study have shown that individuals whose body mass index (BMI) exceeded $27 \mathrm{~kg} / \mathrm{m}^{2}$ at age 20 had a twofold increased risk of developing MS in comparison with subjects with normal body weight. ${ }^{57}$ In a further Swedish study using EIMS and GEMS data, striking associations were observed between BMI status and HLA genotype with regard to MS risk. Obese individuals with the MS-associated HLA genotype (presence of the HLA-DRB $1 * 1501$ allele and absence of the HLA-A*02 allele) had a 16 -fold greater probability of developing MS compared to non-obese individuals without this genetic risk factor. ${ }^{58}$ Other population-based studies have shown that the risk of MS is increased in obese children (aged 7-13 years) ${ }^{60}$ and adolescents (aged 1218 years), ${ }^{61}$ with the effects being most pronounced in girls. As for the possible mechanism of action, it is speculated that a chronic, low-grade inflammatory state can develop as a result of the part played by adipocytes in several metabolic pathways. High levels of leptin, an adipokine secreted by adipose tissue, have been associated with reduced regulatory T-cell activity and increased disease severity in animal models. ${ }^{62,63}$

BMI and MS disease activity or progression. There is also some evidence of a negative impact of obesity on disease-related outcomes in those with MS. A higher BMI has been associated with a poorer response to interferon beta- $1 \mathrm{~b}$ in MS patients. ${ }^{64}$ Moreover, obesity has been associated with increased T1 lesion volume at MRI, ${ }^{65}$ higher disability levels in a cohort of MS subjects who were obese compared with those of normal weight ${ }^{66}$ and in patients at MS diagnosis. ${ }^{67}$

Implications for prevention and patient care. Clearly, educational interventions should not take BMI as the only indicator of health status and should focus more broadly on lifestyle. Adolescents and young adults at high risk of developing MS should be counselled on the need to modify their lifestyle to mitigate their risk. Patients with MS should be encouraged to modify their lifestyle in order to reduce the negative impact of obesity on disease-related outcomes and the risk of associated comorbidities.

\section{Other risk factors}

\section{Salt}

There is some experimental information on the implications of dietary salt on the pathogenesis of CNS autoimmunity. Studies using animal models of MS have shown that high salt diet promotes pathogenic T-cell responses and aggravates autoimmunity, suggesting that high dietary salt intake may promote CNS autoimmunity and increase the risk of MS. ${ }^{68}$ These findings have not as yet been demonstrated in clinical studies, but it would seem prudent for those at high risk of developing MS, and perhaps those already with MS, to moderate salt intake as a lifestyle choice.

\section{Alcohol and caffeine}

The Swedish EIMS and GEMS studies have assessed the possible association between alcohol and caffeine consumption and the risk of developing MS. A dosedependent inverse association between alcohol consumption and MS risk was statistically significant in both men and women. In addition, the detrimental effects of smoking were more pronounced among non-drinkers. ${ }^{69}$ 
Studies in animal models of MS have shown that caffeine may decrease the risk of developing the disease, possibly because of its neuroprotective properties and its role in the suppression of the production of proinflammatory cytokines. ${ }^{70}$ Data from population-based studies showed that the risk of MS was substantially reduced in individuals reporting a high coffee consumption. ${ }^{71}$ However, in two large cohorts of women participating in the Nurse Health Study, the association between MS risk and alcohol or caffeine intake was not confirmed. ${ }^{72}$ Therefore, due to limited research and inconsistent findings, caution is needed in interpreting these results and their significance warrants further investigation.

\section{Stress}

It has been suggested that psychological stress may play a role in the risk of MS, but the evidence in the field is limited and interpretation is difficult due to methodological issues and complexities in the definition and quantification of stress. A number of nationwide studies have been performed in Denmark. In the first study, parents exposed to the loss of a child had an increased risk of MS which was significant only when follow-up was at least 8 years. ${ }^{73}$ In the second study, there was little evidence for a causal association between major stressful life events, such as divorce or the loss of a child or a spouse, and subsequent MS risk. ${ }^{74} \mathrm{~A}$ third study focused on the associations between stressful life events occurring in childhood (parental divorce, parental death, death of a sibling) and subsequent risk of MS reported only weak or modest associations. ${ }^{75}$

As for the potential impact of stress on disease activity, severe stress associated with war increased the frequency of MS relapses. ${ }^{76}$ A meta-analysis of 14 studies showed that non-traumatic stressful life events were associated with an increased risk of disease exacerbation. However, the association between the stressors and MS exacerbation is complex and no firm conclusions can be drawn because measurement of effects was heterogeneous between studies. ${ }^{77}$ It has been postulated that the control of immune function by the major stress systems is impaired in MS and the endogenous inhibiting systems may become inefficient against inflammation. This hypothesis should be tested in prospective epidemiological studies that employ endocrine, immunological and MRI activity markers. ${ }^{78}$

\section{Comorbidities}

Interest in MS-related comorbidities is increasing. Most studies have focused on the presence or impact of comorbidities in patients with MS. Comorbidities have been associated with delays in diagnosis and disability progression. ${ }^{67}$ Modifiable comorbidities arise directly or indirectly from the primary disease (e.g. osteoporosis due to reduced mobility) and their management impacts overall patient outcomes. The interaction between MS and comorbidities is complex. For example, comorbidities may affect decisions on initiation of disease-modifying drugs (DMDs) ${ }^{79}$ and DMDs may increase the risk of certain comorbidities or they may be contraindicated if these comorbidities are already present. ${ }^{80}$ Moreover, common cancers may be diagnosed less frequently in MS patients because of MS-related immunological changes, the adoption of a healthier lifestyle following an MS diagnosis or a delay in cancer detection. ${ }^{67}$

Gaining a better understanding of MS comorbidities may provide insight into the underlying disease processes and promote the health and wellbeing of MS patients.

\section{Implications for patient care}

Comorbidities in MS are finally being given the profile and importance their potential impact warrants. MS neurologists are advised to help prevent comorbidities and to systematically pursue early diagnosis and optimal treatment of modifiable comorbidities, which may produce substantial improvements in patient quality of life. ${ }^{80}$

\section{The gut microbiota-brain axis}

There is growing evidence implicating gut microbiota in the starting phase of several autoimmune diseases. As for MS, the stimuli triggering the autoimmune response have been commonly attributed to environmental factors, in particular microbial infections. However, using the relapsing-remitting mouse model of spontaneously developing experimental autoimmune encephalomyelitis, it has been shown that the commensal gut flora - in the absence of pathogenic agents - is essential in triggering immune processes, leading to a relapsing-remitting autoimmune disease driven by myelin-specific CD4 ${ }^{+}$T cells. ${ }^{81,82}$

Further research is now ongoing to determine the composition of the intestinal microbiota associated with an increased susceptibility to MS, ${ }^{83,84}$ in addition to disease outcomes in those already with $\mathrm{MS},{ }^{85}$ and this research may provide a conceptual basis for exploring new, less invasive treatment strategies. 
Table 2. Achievements and challenges: key points.

The aetiology of MS is multifactorial with both genetic and non-genetic determinants contributing to the risk of the disease

Four environmental factors - EBV infection, low levels of vitamin D, cigarette smoking and increased BMI - are notable for robust evidence supporting their association with the disease

Primary infection with EBV and a history of IM double an individual's risk of MS, with elevated antibody titres to EBV nuclear antigen being observed before the disease onset

Longitudinal studies of vitamin D supplementation and pre-onset serum levels of 25(OH)D support a protective role of vitamin D on MS risk

Cigarette smoking (both active and passive) increases the risk of MS by two to three times

Increased BMI is associated with two- to threefold increased risk of MS and female adolescents are at higher risk Each of the above factors may interact in a complex manner with other genetic and non-genetic determinants Our understanding of the mechanisms by which EBV infection, vitamin D, cigarette smoking and increased BMI influence the risk of MS are limited or mainly speculative and further research is required

There is some - albeit not definitive - evidence that vitamin D and cigarette smoking may also influence the subsequent MS disease course (relapses and/or progression)

MS: multiple sclerosis; EBV: Epstein-Barr virus; BMI: body mass index; IM: infectious mononucleosis; 25(OH)D: 25-hydroxyvita$\min \mathrm{D}_{3}$.

\section{Achievements, gaps in knowledge and future perspectives}

\section{Achievements}

Over the past decades much progress has been made in the identification of genetic and environmental, modifiable risk factors for the onset of MS, including the role of EBV, vitamin D levels, tobacco smoking and BMI (Table 2). These successes have resulted in neurologists being able to provide some guidance on ways to reduce risk in patients' relatives, CIS and RIS subjects and to MS patients regarding lifestyle modification to potentially alter their clinical course.

The literature provides estimates that assist in determining the specific proportion of cases of MS that could be prevented through prevention of the above four major environmental risk factors. ${ }^{86}$ In particular, considering preventive interventions to occur sequentially, and under specific assumptions related to the increased risk associated with each risk factor and the prevalence of exposure in the population, it has been calculated that smoking avoidance would produce $8 \%$, vitamin D supplementation $44 \%$, obesity prevention $15 \%$ and IM prevention $12 \%$ reduction. Together, curtailing these risk factors may prevent over $60 \%$ of MS cases. An EBV vaccine or other intervention that modulates EBV infection or the immune response to EBV could potentially prevent around $90 \%$ of MS cases.

\section{Gaps in knowledge and future perspective}

Most of the existing data concerning established risk factors for MS are derived from RRMS and are naturally dominated by women, so future investigations are warranted in the primary and secondary progressive forms of MS, and in men, that look for modifiable factors to decrease disease risk and possibly ameliorate the subsequent disease course.

Moreover, our understanding of the pathogenic mechanisms underlying the identified risk factors is limited or mainly speculative. An in-depth elucidation of how these factors interfere with the development of MS, or with disease outcomes in those with MS, could lead to new approaches to prevention and treatment.

Future research should also focus on novel, emerging risk factors for which evidence is limited or conflicting to date. To achieve these goals, further efforts should necessarily reinforce the multidisciplinary approach, combining basic and epidemiological research. In this context, there may be an increasing role for 'omic technologies' in elucidating pathogenic mechanisms due to their abilities to give a snapshot of the activity of cells, tissue and organisms at individual points in time. ${ }^{14}$ Future genetic studies may reveal mechanisms that can be modulated by developing novel therapeutic strategies. Epidemiological studies of gene-environment interactions can confirm causal associations, and epidemiological methodology can provide additional evidence of associations suggested by genetic studies. Meta-analyses are powerful ways of combining data and highlighting consistent patterns among studies. It is possible that traditional epidemiological methods may soon become more efficient by the use of social media and mobile technologies in tracking individuals included in epidemiological surveys. ${ }^{87}$ 
Furthermore, a major gap in knowledge is understanding interactions between different genetic and environmental risk factors. Clarifying these interactions and modelling all known risk factors could enable robust 'risk stratification' and, possibly, the evaluation of an 'individualised' risk estimate, in order to identify those individuals who may benefit most from preventive strategies.

Some preliminary attempts at combining risk factors have been undertaken. ${ }^{11}$ The most comprehensive study to date involved the combination of 16 genetic risk loci, sex, and EBV titres into a prediction model. ${ }^{88}$ However, the model fell short of being clinically useful, and more detailed studies of these and additional risk factors in large cohorts are needed to better understand their predictive power in combination.

Finally, there is preliminary work on environmental factors, vitamin D, smoking and obesity in particular, as potential modifiers of the disease course and response to DMDs, ${ }^{89}$ a field that warrants further research.

Overall, although changes in environmental and nutritional factors would surely not eradicate MS completely, they may account for a large number of cases and further progress in this field could have a dramatic impact on the occurrence of the disease. Some of these factors may also impact the subsequent disease course in those who develop MS and hence inform the management of the disease.

\section{Declaration of Conflicting Interests}

The author(s) declared no potential conflicts of interest with respect to the research, authorship and/or publication of this article.

\section{Funding}

The author(s) received no financial support for the research, authorship and/or publication of this article.

\section{References}

1. Hemmer B, Kerschensteiner M and Korn T. Role of the innate and adaptive immune responses in the course of multiple sclerosis. Lancet Neurol 2015; 14: 406-419.

2. Howard J, Trevick S and Younger DS. Epidemiology of multiple sclerosis. Neurol Clin 2016; 34: 919-939.

3. Kalincik $T$ and Bulzkueven H. Observational data: Understanding the real MS world. Mult Scler 2016; 22: $1642-1648$.
4. Ransohoff RM, Hafler DA and Lucchinetti CF. Multiple sclerosis - A quiet revolution. Nat Rev Neurol 2015; 11: 134-142.

5. Dyment DA, Ebers GC and Sadovnick AD. Genetics of multiple sclerosis. Lancet Neurol 2004; 3: 104-110.

6. Sawcer S, Hellenthal G, Pirinen M, et al.; International Multiple Sclerosis Genetics Consortium and Wellcome Trust Case Control Consortium. Genetic risk and a primary role for cell-mediated immune mechanisms in multiple sclerosis. Nature 2011; 476: 214-219.

7. Beecham AH, Patsopoulos NA, Xifara DK, et al.; International Multiple Sclerosis Genetics Consortium. Analysis of immune-related loci identifies 48 new susceptibility variants for multiple sclerosis. Nat Genet 2013; 45: 1353-1360.

8. Ramagopalan SV and Ebers GC. Multiple sclerosis: Major histocompatibility complexity and antigen presentation. Genome Med 2009; 1: gm105.

9. McKay KA, Jahanfar S, Duggan T, et al. Factors associated with onset, relapses or progression in multiple sclerosis: A systematic review. Neurotoxicology. Epub ahead of print 1 April 2016. DOI: 10.1016/j.neuro.2016.03.020.

10. Hillert J and Stawiarz L. The Swedish MS registry - Clinical support tool and scientific resource. Acta Neurol Scand 2015; 132: 11-19.

11. Ramagopalan SV, Dobson R, Meier UC, et al. Multiple sclerosis: Risk factors, prodromes, and potential causal pathways. Lancet Neurol 2010; 9: 727-739.

12. Gilden DH. Infectious causes of multiple sclerosis Lancet Neurol 2005; 4: 195-202.

13. Ascherio A and Munger KL. Environmental risk factors for multiple sclerosis. Part I: The role of infection. Ann Neurol 2007; 61: 288-299.

14. Ascherio A, Munger KL and Lünemann JD. The initiation and prevention of multiple sclerosis. Nat Rev Neurol 2012; 5: 602-612.

15. Goulden R, Ibrahim T and Wolfson C. Is high socioeconomic status a risk factor for multiple sclerosis? A systematic review. Eur J Neurol 2015 22: 899-911.

16. Thacker EL, Mirzaei $F$ and Ascherio A. Infectious mononucleosis and risk for multiple sclerosis: A meta-analysis. Ann Neurol 2006; 59: 499-503.

17. Levin LI, Munger KL, O’Reilly EJ, et al. Primary infection with the Epstein-Barr virus and risk of multiple sclerosis. Ann Neurol 2010; 67: 824-830.

18. Gale CR and Martyn CN. Migrant studies in multiple sclerosis. Prog Neurobiol 1995; 47: 425-448. 
19. Smith $\mathrm{C}$ and Khanna R. The development of prophylactic and therapeutic EBV vaccines. Curr Top Microbiol Immunol 2015; 391: 455-473.

20. Gasperi C, Stüve O and Hemmer B. B cell-directed therapies in multiple sclerosis. Neurodegener Dis Manag 2016; 6: 37-47.

21. Dreyfus DH. Autoimmune disease: A role for new anti-viral therapies? Autoimmun Rev 2011; 11 : 88-97.

22. Marta M, MacManus D, Yousry T, et al. Phase 2 baseline versus treatment clinical trial of the HIV drug raltegravir in patients with active relapsing remitting multiple sclerosis: The INSPIRE study results. Mult Scler 2016; 22(Suppl. 3): 188.

23. Ristori G, Romano S, Cannoni S, et al. Effects of Bacille Calmette-Guerin after the first demyelinating event in the CNS. Neurology 2014; 82: 41-48.

24. Correale J and Farez MF. The impact of environmental infections (parasites) on MS activity. Mult Scler 2011; 17: 1162-1169.

25. Voldsgaard A, Bager P, Garde E, et al. Trichuris suis ova therapy in relapsing multiple sclerosis is safe but without signals of beneficial effect. Mult Scler 2015; 21: $1723-1729$.

26. Smolders J and Damoiseaux J. Vitamin D as a T-cell modulator in multiple sclerosis. Vitam Horm 2011; 86: 401-428.

27. Wergeland S, Torkildsen Ø, Myhr KM, et al. Dietary vitamin D3 supplements reduce demyelination in the cuprizone model. PLoS ONE 2011; 6: e26262.

28. Niino M and Miyazaki Y. Genetic polymorphisms related to vitamin $\mathrm{D}$ and the therapeutic potential of vitamin D in multiple sclerosis. Can J Physiol Pharmacol 2015; 93: 319-325.

29. Munger KL, Åivo J, Hongell K, et al. Vitamin D status during pregnancy and risk of multiple sclerosis in offspring of women in the Finnish Maternity Cohort. JAMA Neurol 2016; 73: 515-519.

30. Mokry LE, Ross S, Ahmed OS, et al. Vitamin D and risk of multiple sclerosis: A Mendelian randomization study. PLoS Med 2015; 12: e1001866.

31. Rhead B, Bäärnhielm M, Gianfrancesco M, et al. Mendelian randomization shows a causal effect of low vitamin D on multiple sclerosis risk. Neurol Genet 2016; 2: e97.

32. Burton JM, Kimball S, Vieth R, et al. A phase I/II dose-escalation trial of vitamin D3 and calcium in multiple sclerosis. Neurology 2010; 74: 1852-1859.

33. Kimball S, Vieth R, Dosch HM, et al. Cholecalciferol plus calcium suppresses abnormal PBMC reactivity in patients with multiple sclerosis. J Clin Endocrinol Metab 2011; 96: 2826-2834.
34. Soilu-Hänninen M, Aivo J, Lindström BM, et al. A randomised, double blind, placebo controlled trial with vitamin D3 as an add on treatment to interferon beta- $1 \mathrm{~b}$ in patients with multiple sclerosis. $J$ Neurol Neurosurg Psychiatry 2012; 83: 565-571.

35. Kampman MT, Steffensen LH, Mellgren SI, et al. Effect of vitamin D3 supplementation on relapses, disease progression, and measures of function in persons with multiple sclerosis: Exploratory outcomes from a double-blind randomised controlled trial. Mult Scler 2012; 18: 1144-1151.

36. Stein MS, Liu Y, Gray OM, et al. A randomized trial of high-dose vitamin D2 in relapsing-remitting multiple sclerosis. Neurology 2011; 77: 1611-1618.

37. Mosayebi G, Ghazavi A, Ghazami K, et al. Therapeutic effect of vitamin D3 in multiple sclerosis patients. Immunol Invest 2011; 40: 627-639.

38. Smolders J, Hupperts R, Barkhof F, et al. Efficacy of vitamin D3 as add-on therapy in patients with relapsing-remitting multiple sclerosis receiving subcutaneous interferon beta-1a: A Phase II, multicenter, double-blind, randomized, placebocontrolled trial. J Neurol Sci 2001; 311: 44-49.

39. Bhargava P, Cassard S, Steele SU, et al. The vitamin $\mathrm{D}$ to ameliorate multiple sclerosis (VIDAMS) trial: Study design for a multicenter, randomized, double-blind controlled trial of vitamin D in multiple sclerosis. Contemp Clin Trials 2014; 39: 288-293.

40. Dörr J, Ohiraun S, Skarabis H, et al. Efficacy of vitamin D supplementation in multiple sclerosis (EVIDIMS Trial): Study protocol for a randomized controlled trial. Trials 2012; 13: 15.

41. MS Research Australia. Vitamin D MS prevention trial: Update 22 June 2016, http://www.msra.org.au/ vitamin-d-ms-prevention-trial-update (accessed 29 July 2016).

42. Ascherio A, Munger KL, White R, et al. Vitamin D as an early predictor of multiple sclerosis activity and progression. JAMA Neurol 2014; 71: 306-314.

43. Fitzgerald KC, Munger KL, Köchert K, et al. Association of vitamin D levels with multiple sclerosis activity and progression in patients receiving interferon beta-1b. JAMA Neurol 2015; 72: 14581465 .

44. Handel AE, Williamson AJ, Disanto G, et al. Smoking and multiple sclerosis: An updated metaanalysis. PLos ONE 2011; 6: e16149.

45. Hedström AK, Hillert J, Olsson T, et al. Smoking and multiple sclerosis susceptibility. Eur J Epidemiol 2013; 28: 767-774.

46. Salzer J, Hallmans G, Nyström M, et al. Smoking as a risk factor for multiple sclerosis. Mult Scler 2013; 19 : 1022-1027. 
47. Palacios N, Alonso A, Brønnum-Hansen H, et al. Smoking and increased risk of multiple sclerosis: Parallel trends in the sex ratio reinforce the evidence. Ann Epidemiol 2011; 21: 536-542.

48. Di Pauli F, Reindl M, Ehling R, et al. Smoking is a risk factor for early conversion to clinically definite multiple sclerosis. Mult Scler 2008; 14: 1026-1030.

49. Manouchehrinia A, Tench CR, Maxted J, et al. Tobacco smoking and disability progression in multiple sclerosis: United Kingdom cohort study. Brain 2013; 136: 2298-2304.

50. Ramanujam R, Hedström AK, Manouchehrinia A, et al. Effect of smoking cessation on multiple sclerosis prognosis. JAMA Neurol 2015; 72: 1117 1123.

51. Barragán-Martinez C, Speck-Hernández CA, Montoya-Ortiz G, et al. Organic solvents as risk factor for autoimmune diseases: A systematic review and meta-analysis. PLoS ONE 2012; 7: e51506.

52. Hedström AK, Bäärnhielm M, Olsson T, et al. Tobacco smoking, but not Swedish snuff use, increases the risk of multiple sclerosis. Neurology 2009; 73: 696-701.

53. Odoardi F, Sie C, Streyl K, et al. T cells become licensed in the lung to enter the central nervous system. Nature 2012; 488: 675-679.

54. Munger KL, Chitnis T and Ascherio A. Body size and risk of MS in two cohorts of US women. Neurology 2009; 73: 1543-1550.

55. Wesnes K, Riise T, Casetta I, et al. Body size and the risk of multiple sclerosis in Norway and Italy: The EnvIMS study. Mult Scler 2015; 21: 388-395.

56. Gianfrancesco MA, Acuna B, Shen L, et al. Obesity during childhood and adolescence increases susceptibility to multiple sclerosis after accounting for established genetic and environmental risk factors. Obes Res Clin Pract 2014; 8: e435-e447.

57. Hedström AK, Olsson T and Alfredsson L. High body mass index before age 20 is associated with increased risk for multiple sclerosis in both men and women. Mult Scler 2012; 18: 1334-1336.

58. Hedström AK, Lima Bomfim I, Barcellos L, et al. Interaction between adolescent obesity and HLA risk genes in the etiology of multiple sclerosis. Neurology 2014; 82: 865-872.

59. Ponsonby AL, Lucas RM, Dear K, et al. The physical anthropometry, lifestyle habits and blood pressure of people presenting with a first clinical demyelinating event compared to controls: The Ausimmune study. Mult Scler 2013; 19: 1717-1725.

60. Munger KL, Bentzen J, Laursen B, et al. Childhood body mass index and multiple sclerosis risk: A longterm cohort study. Mult Scler 2013; 19: 1323-1329.
61. Langer-Gould A, Brara SM, Beaber BE, et al. Childhood obesity and risk of pediatric multiple sclerosis and clinically isolated syndrome. Neurology 2013; 80: 548-552.

62. Matarese G, Carrieri PB, La Cava A, et al. Leptin increase in multiple sclerosis associates with reduced number of CD4(+)CD25+ regulatory T cells. Proc Natl Acad Sci U S A 2005; 102: 5150-5155.

63. Matarese G, Di Giacomo A, Sanna V, et al. Requirement for leptin in the induction and progression of autoimmune encephalomyelitis. $J$ Immunol 2001; 166: 5909-5918.

64. Kvistad SS, Myhr KM, Holmøy T, et al. Body mass index influence interferon-beta treatment response in multiple sclerosis. J Neuroimmunol 2015; 288: 92-97.

65. Kappus N, Weinstock-Guttman B, Hagemeier J, et al. Cardiovascular risk factors are associated with increased lesion burden and brain atrophy in multiple sclerosis. J Neurol Neurosurg Psychiatry 2016; 87: 181-187.

66. Tettey P, Siejka D, Simpson S, Jr, et al. Frequency of comorbidities and their association with clinical disability and relapse in multiple sclerosis. Neuroepidemiology 2016; 46: 106-113.

67. Marrie RA, Horwitz R, Cutter G, et al. Comorbidity delays diagnosis and increases disability at diagnosis in MS. Neurology 2009; 72: 117-124.

68. Hucke S, Wiendl $\mathrm{H}$ and Klotz L. Implications of dietary salt intake for multiple sclerosis pathogenesis. Mult Scler 2016; 22: 133-139.

69. Hedström AK, Hillert J, Olsson T, et al. Alcohol as a modifiable lifestyle factor affecting multiple sclerosis risk. JAMA Neurol 2014; 71: 300-305.

70. Wang T, Xi NN, Chen Y, et al. Chronic caffeine treatment protects against experimental autoimmune encephalomyelitis in mice: Therapeutic window and receptor subtype mechanism. Neuropharmacology 2014; 86: 203-211.

71. Hedström AK, Mowry EM, Gianfrancesco MA, et al. High consumption of coffee is associated with decreased multiple sclerosis risk; results from two independent studies. J Neurol Neurosurg Psychiatry 2016; 87: 454-460.

72. Massa J, O'Reilly EJ, Munger KL, et al. Caffeine and alcohol intakes have no association with risk of multiple sclerosis. Mult Scler 2013; 19: 53-58.

73. Li J, Johansen C, Brønnum-Hansen H, et al. The risk of multiple sclerosis in bereaved parents: A nationwide cohort study in Denmark. Neurology 2004; 62: 726-729.

74. Nielsen NM, Bager P, Simonsen J, et al. Major stressful life events in adulthood and risk of multiple 
sclerosis. J Neurol Neurosurg Psychiatry 2014; 85: 1103-1108.

75. Nielsen NM, Pedersen BV, Stenager E, et al. Stressfu life-events in childhood and risk of multiple sclerosis: A Danish nationwide cohort study. Mult Scler 2014; 20: 1609-1615.

76. Golan D, Somer E, Dishon S, et al. Impact of exposure to war stress on exacerbations of multiple sclerosis. Ann Neurol 2008; 64: 143-148.

77. Mohr DC, Hart SL, Julian L, et al. Association between stressful life events and exacerbation in multiple sclerosis: A meta-analysis. BMJ 2004; 328: 731.

78. Gold SM, Mohr DC, Huitinga I, et al. The role of stress-response systems for the pathogenesis and progression of MS. Trends Immunol 2005; 26: 644-652.

79. Zhang T, Tremlett H, Leung S, et al. Examining the effects of comorbidities on disease-modifying therapy use in multiple sclerosis. Neurology 2016; 86: $1287-1295$.

80. Berrigan LI, Fisk JD, Patten SB, et al. Health-related quality of life in multiple sclerosis: Direct and indirect effects of comorbidity. Neurology 2016; 86: $1417-1424$.

81. Berer K, Mues M, Koutrolos M, et al. Commensal microbiota and myelin autoantigen cooperate to trigger autoimmune demyelination. Nature 2011; 479: $538-541$

82. Lee YK, Menezes JS, Umesaki Y, et al. Proinflammatory T-cell responses to gut microbiota promote experimental autoimmune encephalomyelitis. Proc Natl Acad Sci U S A 2011; 108(Suppl. 1): 4615-4622.

83. Jangi S, Gandhi R, Cox LM, et al. Alterations of the human gut microbiome in multiple sclerosis. Nat Commun 2016; 7: 12015.

84. Chen J, Chia N, Kalari KR, et al. Multiple sclerosis patients have a distinct gut microbiota compared to healthy controls. Sci Rep 2016; 6: 28484.

85. Tremlett H, Fadrosh DW, Faruqi AA, et al. Gut microbiota composition and relapse risk in pediatric MS: A pilot study. J Neurol Sci 2016; 363: 153-157.

86. Ascherio A and Munger KL. Epidemiology of multiple sclerosis: From risk factors to prevention An update. Semin Neurol 2016; 36: 103-114.

87. Van der Mei I, Lucas RM, Yaylor BV, et al. Population attributable fractions and joint effects of key risk factors for multiple sclerosis. Mult Scler 2016; 22: 461-469.

88. De Jager PL, Chibnik LB, Cui J, et al. Integration of genetic risk factors into a clinical algorithm for multiple sclerosis susceptibility: A weighted genetic risk score. Lancet Neurol 2009; 8: 1111-1119.

89. Petersen ER, Oturai AB, Koch-Henriksen N, et al. Effect of smoking, a NAT1 genetic variant and HLA genes on disease activity in MS patients before and during interferon-beta treatment. Mult Scler 2016; 22(Suppl. 3): 58.

\section{Appendix 1}

\section{The 2016 ECTRIMS Focused Workshop \\ Group}

Lars Alfredsson, Institute of Environmental Medicine, Karolinska Institute, Stockholm, Sweden.

Francesca Aloisi, Department of Cell Biology and Neuroscience, Istituto Superiore di Sanità, Rome, Italy.

Maria Pia Amato, Department of NEUROFARBA, Section Neurosciences, University of Florence, Florence, Italy.

Alberto Ascherio, Departments of Epidemiology and Nutrition, Harvard T.H. Chan School of Public Health, Boston, MA, USA; Channing Laboratory, Department of Medicine, Brigham and Women's Hospital, Boston, MA, USA; Harvard Medical School, Boston, MA, USA.

Elisa Baldin, Epidemiology and Biostatistics Service, IRCCS Institute of Neurological Sciences of Bologna, Bologna, Italy.

Kjetil Bjørnevik, Department of Global Public Health and Primary Care, University of Bergen, Bergen, Norway; Norwegian Multiple Sclerosis Competence Centre, Department of Neurology, Haukeland University Hospital, Bergen, Norway.

Manuel Comabella, Department de NeurologíaNeuroinmunología, Centre d'Esclerosi Múltiple de Catalunya, Vall d'Hebron Institut de Recerca, Hospital Universitari Vall d'Hebron, Universitat Autònoma de Barcelona, Barcelona, Spain/Red Española de Esclerosis Múltiple (REEM).

Jorge Correale, Department of Neurology, Institute for Neurological Research Dr. Raúl Carrea, FLENI, Buenos Aires, Argentina.

Marianna Cortese, Department of Global Public Health and Primary Care, University of Bergen, Bergen, Norway; Norwegian Multiple Sclerosis Competence Centre, Department of Neurology, Haukeland University Hospital, Bergen, Norway.

Tobias Derfuss, Departments of Neurology and Biomedicine, University Hospital Basel, Basel, Switzerland. 
Marie D’Hooghe, Center for Neurosciences, Vrije Universiteit Brussel (VUB), Laarbeeklaan 101, 1090 Brussels, Belgium.

Angelo Ghezzi, Gallarate Hospital, Gallarate (Varese), Italy.

Julian Gold, Queen Mary University of London, London, UK.

Kerstin Hellwig, Department of Neurology, St. JosefHospital, Ruhr-University Bochum, Bochum, Germany.

Bernhard Hemmer, Department of Neurology, Klinikum rechts der Isar, Technische Universität München, Munich, Germany.

Nils Koch-Henricksen, The Danish Multiple Sclerosis Registry, Department of Neurology, Rigshospitalet, Copenhagen, Denmark; Department of Clinical Epidemiology, Clinical Institute, Aarhus University, Olof Palmes Allé 43-45, 8200, Aarhus N, Denmark.

Annette Langer Gould, Kaiser Permanente Southern California, Pasadena, CA, USA.

Roland Liblau, Centre de Physiopathologie ToulousePurpan, CPTP, Université de Toulouse, CNRS, Inserm, UPS, Toulouse, France.

Ralf Linker, Department of Neurology, Friedrich-Alexander University Erlangen-Nürnberg, Erlangen, Germany.

Francesco Lolli, Dipartimento di Scienze Biomediche, Sperimentali e Cliniche 'Mario Serio', Florence, Italy.

Robyn Lucas, National Centre for Epidemiology \& Population Health, Research School of Population Health, The Australian National University, Canberra, ACT, Australia.

Jan Lünemann, Department of Neuroinflammation, Institute of Experimental Immunology, University of Zürich, Switzerland; Department of Neurology, University Hospital Zürich, Zürich, Switzerland.

Melinda Magyari, Department of Neurology, Danish Multiple Sclerosis Center, Rigshospitalet, University of Copenhagen, Blegdamsvej 9, 2100, Copenhagen, Denmark.

Luca Massacesi, Department of NEUROFARBA, Section Neurosciences, University of Florence, Florence, Italy.

Ariel Miller, Division of Neuroimmunology \& Multiple Sclerosis Center, Lady Davis Carmel Medical Center, Haifa, Israel; Ruth and Bruce Rappaport Faculty of Medicine, Technion - Israel Institute of Technology, Haifa, Israel.
David H Miller, Queen Square MS Centre, Department of Neuroinflammation, Institute of Neurology, University College London, London, UK.

XavierMontalban, ServeideNeurologia-Neuroimmunologia, Centre d'Esclerosi Múltiple de Catalunya, Vall d'Hebron, Barcelona, Spain.

Philippe Monteyne, GlaxoSmithKline Biologicals S.A., Rixensart, Belgium.

Ellen Mowry, Department of Neurology (E.M.M.), Johns Hopkins, Baltimore, MD, USA

Christian Münz, Viral Immunobiology, Institute of Experimental Immunology, University of Zürich, Zürich, Switzerland.

Nete M Nielsen, Department of Epidemiology Research, Statens Serum Institut, Copenhagen, Denmark.

Tomas Olsson, Neuroimmunology Unit, Department of Clinical Neuroscience, Center for Molecular Medicine, Karolinska Institutet, Stockholm, Sweden.

Celia Oreja-Guevara, Department of Neurology, Institute of Neurosciences, Hospital Clínico San Carlos, San Carlos Institute for Health Research (IdISSC), Universidad Complutense de Madrid, Madrid, Spain.

Susana Otero, MS Centre of Catalonia (Cemcat), Vall d'Hebron University Hospital, Barcelona, Spain.

Maura Pugliatti, Department of Biomedical and Specialty Surgical Sciences, Section of Clinical Neurology, University of Ferrara, Ferrara, Italy.

Stephen Reingold, Scientific and Clinical Review Associates, LLC, Salisbury, CT, USA.

Trond Riise, Department of Global Public Health and Primary Care, University of Bergen, Bergen, Norway; Norwegian MS Competence Center, Haukeland University Hospital, Bergen, Norway.

Neil Robertson, Division of Psychological Medicine and Clinical Neurosciences, Cardiff University, Cardiff, UK.

Marco Salvetti, Centre for Experimental Neurological Therapies (CENTERS), S Andrea Hospital Site, Sapienza University, Rome, Italy.

Youssef Sidhom, Department of Neurology, PitiéSalpêtrière Hospital, Paris, France; Department of Neurology, Razi Hospital, Tunis, Tunisia.

Joost Smolders, Academic MS Center Limburg, Zuyderland Medical Center, Sittard, The Netherlands; MS Center CWZ, 
Department of Neurology, Canisius Wilhelmina Hospital, Nijmegen, The Netherlands.

Per Soelberg Sørensen, Danish Multiple Sclerosis Center, Department of Neurology, Rigshospitalet, University of Copenhagen, Copenhagen, Denmark.

Ludvig Sollid, Institute of Transplantation Immunology, National Hospital and University of Oslo, Oslo, Norway.

Israel Steiner, Department of Neurology, Rabin Medical Center, Campus Beilinson, 49100, Petah Tikva, Israel.

Egon Stenager, Department of Regional Health Research, University of Southern Denmark, Odense, Denmark; MS-Clinic of Southern Jutland (Sønderborg, Vejle, Esbjerg), Department of Neurology, Sønderborg Hospital, Sønderborg, Denmark.

Peter Sundstrom, Department of Pharmacology and Clinical Neuroscience, Section of Neurology, Umeå University, Umeå, Sweden.
Bruce V Taylor, Menzies Institute for Medical Research, University of Tasmania, Hobart, TAS, Australia.

Helen Tremlett, Faculty of Medicine (Neurology), Djavad Mowafaghian Centre for Brain Health, Vancouver Coastal Health Research Institute, University of British Columbia, Vancouver, BC, Canada.

Maria Trojano, Neurosciences and Sense Organs, University of Bari Aldo Moro, Bari, Italy.

Antonio Uccelli, University of Genova, DINOGMI, Genova, Italy.

Emmanuelle Waubant, Department of Neurology, University of California - San Francisco, San Francisco, CA, USA.

Hartmut Wekerle, Hertie Senior Professor Group Neuroimmunology, Max Planck Institute of Neurobiology, Martinsried, Germany; Munich Cluster for Systems Neurology (SyNergy), Ludwig-Maximilians-Universität München, Munich, Germany.
Visit SAGE journals online journals.sagepub.com/ home/msj

(SAGE journals 